\title{
Autologous Fat Transfer in a Patient with Lupus Erythematosus Profundus
}

\author{
Jimi Yoon ${ }^{\mathrm{a}} \mathrm{Hwa} \mathrm{Mi} \mathrm{Kim}^{\mathrm{a}}$ Tae-Heung Kim ${ }^{\mathrm{b}}$ \\ Chung-Won Kim ${ }^{c}$ Young-Woo Sun ${ }^{b}$ Tae-Jin Yoon ${ }^{a}$ \\ ${ }^{a}$ Department of Dermatology and Institute of Health Sciences, School of \\ Medicine, Gyeongsang National University, Jinju, ${ }^{b}$ White-Line Skin Clinic and \\ Research Center, and 'Department of Dermatology, Changwon Fatima Hospital, \\ Changwon, Korea
}

\section{Key Words}

Lupus erythematosus profundus · Fat transfer

\begin{abstract}
Lupus erythematosus profundus, a form of chronic cutaneous lupus erythematosus, is a rare inflammatory disease involving in the lower dermis and subcutaneous tissues. It primarily affects the head, proximal upper arms, trunk, thighs, and presents as firm nodules, 1 to $3 \mathrm{~cm}$ in diameter. The overlying skin often becomes attached to the subcutaneous nodules and is drawn inward to produce deep, saucerized depressions. We present a rare case of lupus erythematosus profundus treated with autologous fat transfer.
\end{abstract}

\section{Introduction}

Lupus erythematosus (LE) profundus, a form of chronic cutaneous lupus erythematosus, is a rare inflammatory disease involving in the lower dermis and subcutaneous tissues. It primarily affects the head, proximal upper arms, trunk, thighs, and presents as firm nodules, 1 to $3 \mathrm{~cm}$ in diameter. The overlying skin often becomes attached to the subcutaneous nodules and is drawn inward to produce deep, saucerized depressions. We present a rare case of LE profundus treated with autologous fat transfer.

\section{Case Report}

A 25-year-old woman was referred for consultation of treating a 7-year history of enlarging atrophic lesions on the right side of the temple area. The patient denied any congenital deformity 
from her birth or direct trauma on this lesion. Physical examination revealed a $5.5 \times 7 \mathrm{~cm}$ sized, depressed, slightly pigmented patch on the right temple area (fig. 1a). She did not complain of general symptoms such as photosensitivity, oral ulcer, arthritis, or Raynaud phenomenon. Laboratory findings revealed decreased level of leukocytes $\left(3.8 \times 10^{3} / \mu \mathrm{l}\right)$, elevated level of rheumatoid factor (53.6 $\mathrm{IU} / \mathrm{ml}$ ), and positive antinuclear antibody (titer 1:160). But the levels of complement 3 , complement 4 , lupus anticoagulant and Coomb's test were all within normal range. Neurological and radiographical examination showed no evidence of neuromuscular paralysis or bony abnormalities. Histopathologically, perivascular fibrosis and predominantly lobular lymphohistiocytic infiltrate, fat necrosis, and hyalinization of adipose lobules were prominent (fig. 2). Based on the above clinical and histopathological findings, we diagnosed it as LE profundus. The patient was treated with prednisolone $(10 \mathrm{mg} /$ day) and intralesional injection of triamcinolone acetonide $(20 \mathrm{mg} / \mathrm{ml})$ weekly for 3 months. The atrophic lesion improved and became softer and showed no growth of size. We performed fat transfer retrieving usable fat from both of her flanks. The operation was performed under the local tumescent anesthesia. Once the entry sites on her flank were determined, an aspiration cannula (Wooju Medical Instrument Co., Korea) for the fat graft was attached to a $10-\mathrm{ml}$ Luer-lock syringe (Becton Dickinson, Singapore) filled with the tumescent solution (0.5 ml of 1:1,000 epinephrine, $15 \mathrm{ml}$ of $2 \%$ lidocaine, $2.5 \mathrm{ml}$ of $10 \%$ sodium bicarbonate and $500 \mathrm{ml}$ of physiologic saline solution). The long cannula was placed through the stab incision and directed out from the injection site in a fanlike pattern. We aspirated $60 \mathrm{ml}$ of fat via the $10-\mathrm{ml}$ Luer-lock syringe. We conducted a fresh fat transfer for correction of her facial defect. The remaining fat was spared for future frozen fat grafts. The residual fat was put in the 10-ml Luer-lock syringe and wrapped in the parafilm sterilized with EO gas, then packed in the sterile plastic bag and finally stored in a domestic freezer at $-18^{\circ} \mathrm{C}$ for future frozen fat transfer. For fat transfer, the aspirated fat was mixed with normal saline and left in the 30-mm-sized wirenet test tube for $5 \mathrm{~min}$. Once the blood and the impurities were removed and the fat was washed with normal saline 5 times, it was kept at room temperature for $10 \mathrm{~min}$. We transferred this concentrated fat from the 10 -ml syringes into individual 1-ml syringes (Becton Dickinson, Singapore) using a Luer-lock transfer device. The stab incision was made just lateral to the infraorbital rim. The 18-gauge blunt cannula was injected into the cavities above the periosteum of the temple and forehead. The fat was injected via a bended 18-gauge NoKor needle (Becton Dickinson, Singapore) at many different angles. After the conclusion of fat injections, the face was cleansed with isotonic sodium chloride solution and a small amount of antibiotic ointment was placed on the stab incisions. The injected areas were aggressively iced for the first $48 \mathrm{~h}$ to decrease edema and ecchymoses. The operation provided an impressive cosmetic benefit to the patient and no complication and failure of grafts occurred. For the large depressed area, four time frozen fat transfers were additionally performed at the interval of 1.5 to 2 months. The improvement maintained stable for the 6-month follow-up.

\section{Discussion}

LE profundus, a form of chronic cutaneous LE, is a rare inflammatory disease involving in the lower dermis and subcutaneous tissues [1]. It primarily affects the head, proximal upper arms, trunk, thighs, and presents as firm nodules, 1 to $3 \mathrm{~cm}$ in diameter. The overlying skin often becomes attached to the subcutaneous nodules and is drawn inward to produce deep, saucerized depressions [2]. The histopathologic examination reveals a deep lymphocytic infiltration in the fat lobules and in the septa. A distinctive feature is the 'hyaline necrosis' of the fat, in which portions of the fat lobule have lost nuclear staining of the fat cells and have an accumulation of fibrin and other proteins in a homogenous eosinophilic matrix between the residual fat cells and extracellular fat globules [2]. Roughly 50 percent of patients with LE profundus have evidence of systemic LE, but the systemic features tend to be less severe [2]. As shown in our case, autologous fat transfer is a good choice to correct atrophic lesion when LE profundus manifests as hemifacial atrophy of the skin and subcutaneous tissue. Autologous fat transfer is widely performed by dermatologists for facial augmentation and rejuvenation in cosmetic dermatology. It has no allergic reaction or rejection via 
immune process because it is autograft with the same human leukocyte antigen. Fat tissue as dermal filler is plentiful and easy to harvest, and it is completely biocompatible and has a proven potential for lasting viability [3]. The high rate of adipose-derived stem cells in fat tissue confers soft-tissue regeneration properties, especially for radio dermatitis, atrophic scars, chronic ulcerations and antiageing therapy [3]. Recent evidences support the utility of adipocytes for a potential stem cell as well as collagen stimulation [4]. In autologous fat transfer, the local milieu remains more stable presumably due to adipose-derived stem cells [5]. Adipose-derived stem cells may change fibrous tissues and blood vessels when there is an increase in the volume of adipose tissues or subcutaneous tissues [5]. But autologous fat transfer is not as commonly used in connective tissue diseases because of dermatologists' unwillingness to treat advanced connective tissue diseases. We present a rare case of LE profundus treated with autologous fat transfer.
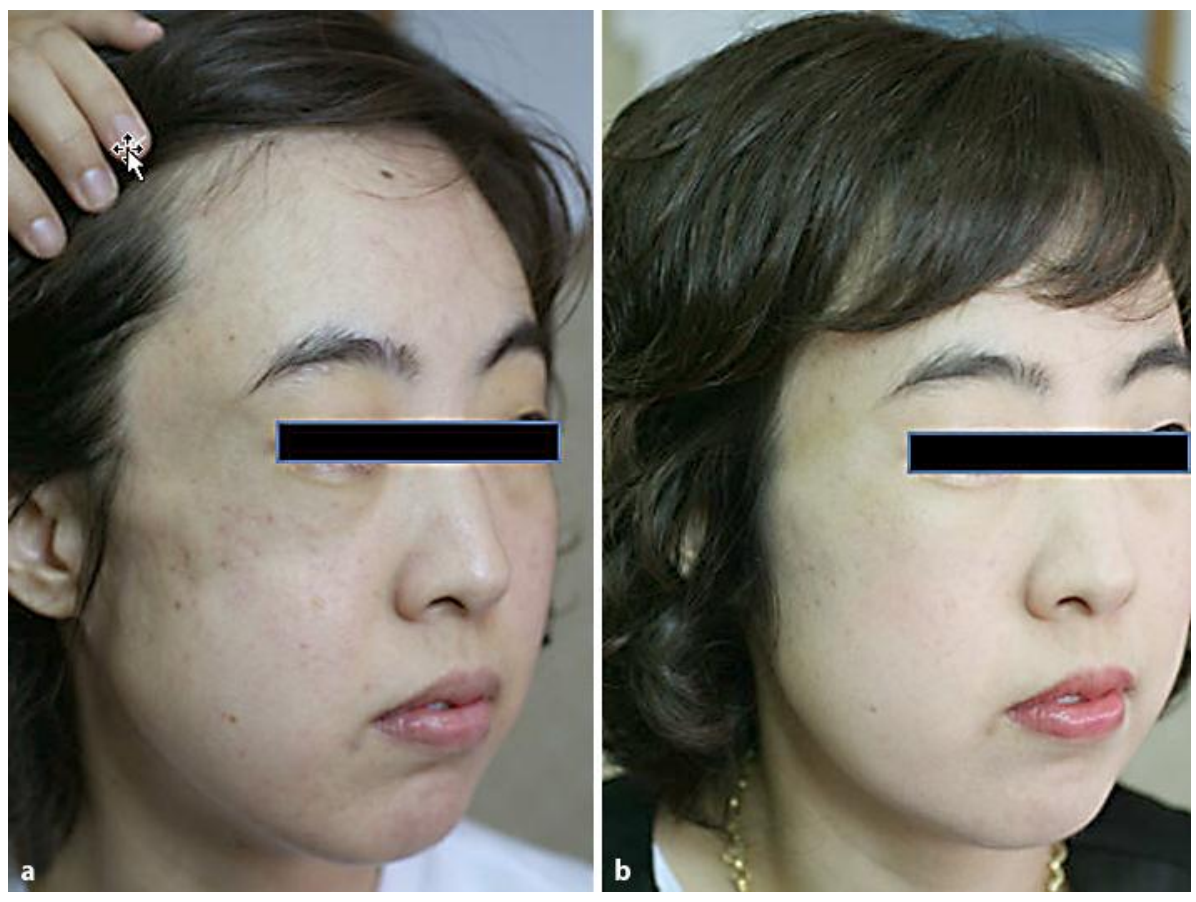

Fig. 1. a A $5.5 \times 7 \mathrm{~cm}$ sized diffuse depressed patch on the right side of the temple area. $\mathbf{b}$ The patient 6 months after autologous fat transfer. There was some improvement cosmetically, making the patient's atrophic lesions more even. 


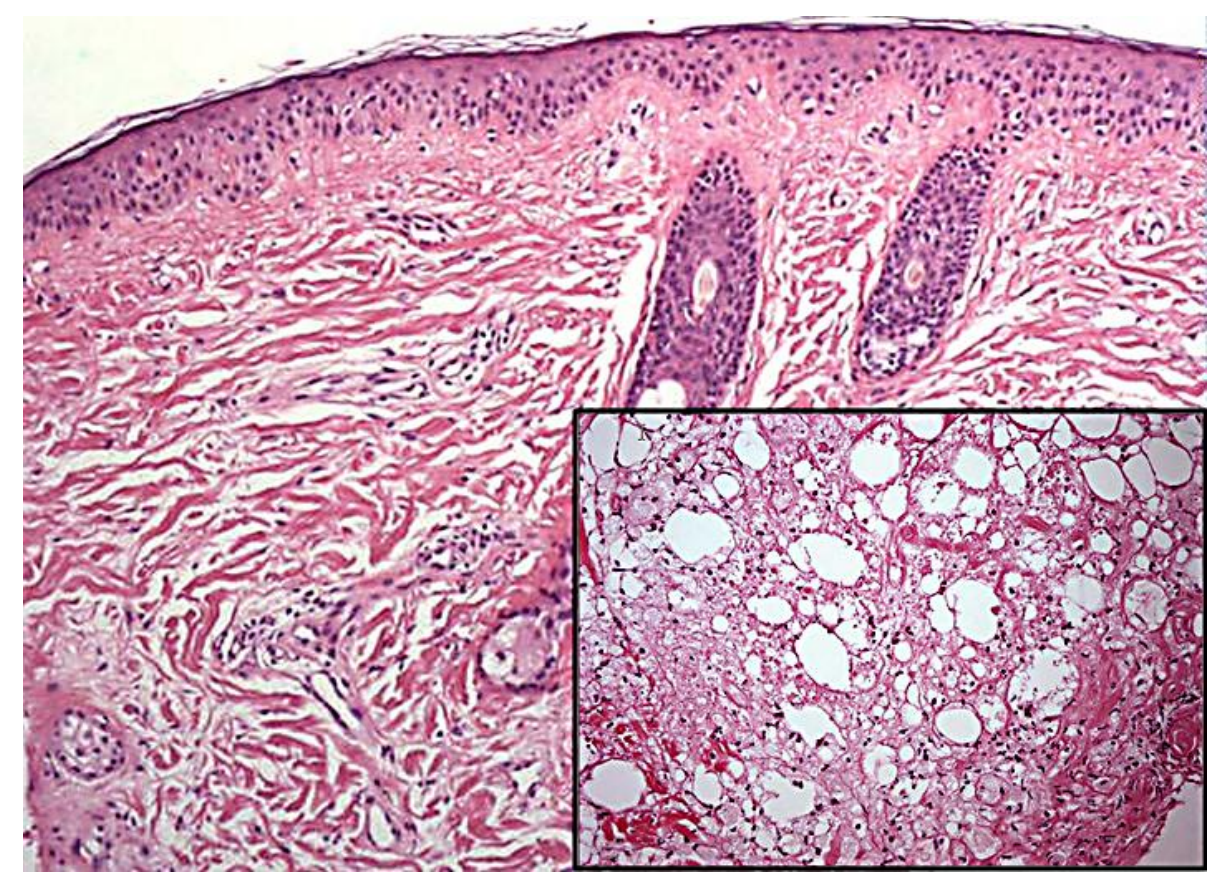

Fig. 2. The histopathologic findings showed a perivascular fibrosis $(\mathrm{HE}, \times 12.5)$. In the inset, lobular lymphohistiocytic infiltrate and fat necrosis are prominent. Also the hyalinization of adipose tissues between the fat cells and extracellular fat globules is evident, showing a homogenous eosinophilic matrix (HE, ×400).

\section{References}

1 Goldsmith K, Katz S, Gilhrest B, et al: Dermatology in General Medicine. 8th ed; Philadelphia, McGraw Hill, 1917, p 155.

$\checkmark 2$ Watanabe T, Tsuchida T: Classification of lupus erythematosus based upon cutaneous manifestations; dermatological, systemic and laboratory findings in 191 patients. Dermatol 1995;190:277-283.

3 Nguyen PS, Desouches C, Gay AM, et al: Development of micro-injection as an innovative autologous fat graft technique: The use of adipose tissue as dermal filler. J Plast Reconstr Aesthet Surg 2012 [Epub ahead of print].

4 Pinski KS, Coleman WP: Microlipoinjection and autologous collagen. Dermatol Clin 1995;13:339-351.

5 Coleman SR: Structural fat grafting: more than a permanent filler. Plast Reconstr Surg 2006;118(suppl):108S-120S. 